\title{
La presencia de Estados Unidos en la formación de los militares colombianos a mediados del siglo $\mathrm{XX}^{*}$
}

\author{
Adolfo León Atehortúa Cruz Ph. D.*
}

\begin{abstract}
Resumen
El presente artículo da cuenta de la influencia ejercida por los Estados unidos en el ejército colombiano en la mitad del siglo XX. Esta influencia se manifestó en el envío de armamento a nuestro país, la instrucción de soldados colombianos en terreno norteamericano y los cambios introducidos en diferentes unidades militares de acuerdo a parámetros estadounidenses. La cooperación militar entre estos dos países se fortaleció a partir de episodios como la defensa del Canal de Panamá y la Guerra de Corea. Es fundamental observar cómo esta alianza fue un signo de la importancia geográfica, estratégica y política que Colombia ha tenido para los intereses de Estados Unidos, y los aspectos ideológicos involucrados en esta relación.
\end{abstract}

Palabras claves: Ejército colombiano, Estados Unidos, cooperación militar, política exterior.

\begin{abstract}
This article discusses the influence of the United States in the Colombian army in the middle of the twentieth century. This influence is manifested in the shipment of weapons, training of Colombian troops on American field and changes in military units according to U.S. standards. Military cooperation between the two countries was strengthened from episodes like the defense of the Panama Canal and the Korean War. Is essential to examine how this alliance was a sign of the geographical, strategic and political importance that Colombia has for U.S. interests, and ideological aspects involved in this relation.
\end{abstract}

Keywords: Colombian army, U.S., military cooperation, foreign policy.

\section{HISTORIA DE UN ACERCAMIENTO. DE SANTOS A LA GUERRA DE COREA}

El acercamiento político y militar entre Colombia y Estados Unidos se selló definitivamente ante los peligros de una confrontación mundial y la necesidad de

\footnotetext{
*Articulo tipo 2: de reflexión según Colciencias.

** Licenciado en historia de la Universidad del Valle, magister en historia de la Universidad Nacional de Colombia y Doctor en Sociología de la Escuela de Altos Estudios de Ciencias Sociales de París. Profesor Titular Departamento de Ciencias Sociales, Decano Facultad de Humanidades Universidad Pedagógica Nacional.
} 
defender el Canal de Panamá a finales de los años treinta del siglo veinte. Atrás quedó la afrenta por el Istmo y el rechazo a la intromisión estadounidense en territorio colombiano; atrás quedó la oposición y la negativa para un acuerdo que desagraviara lo perdido. El "respice polum" y la "política del buen vecino" coparon la escena de uno y otro lado.

A lo largo de la Segunda Guerra Mundial y más aún, después de ella, la proximidad de Colombia y de América Latina con la potencia del norte fue mucho más estrecha. Estados Unidos comprendió la importancia estratégica, económica y militar de los países al sur del río Bravo y, en especial, aquellos cuya ubicación geográfica resultaba vital para sus intereses. Estados Unidos estructuró, entonces, una nueva diplomacia basada en la "no intervención", en los acuerdos comerciales y en los préstamos de ayuda; pero, en lo fundamental, su política se dirigió a conquistar la amistad de las fuerzas militares y a lograr su compromiso de lealtad en caso de un ataque extracontinental. En lo que respecta a Colombia, el interés prioritario de Estados Unidos persiguió la estabilidad política, la adhesión del país a sus objetivos comerciales y de mercado, la garantía irrestricta de los gobiernos para luchar a su lado en defensa del Canal de Panamá y del Caribe, y la disposición de las fuerzas militares para obrar como apoyo y retaguardia.

Tras este último propósito, la actividad de Estados Unidos no escatimó esfuerzos. A partir de la contratación de las misiones naval y aérea en época del presidente Eduardo Santos (1938-1942), los acercamientos directos entre las fuerzas armadas norteamericanas y las colombianas se sucedieron con repetida frecuencia. La relación no se interrumpió con el segundo gobierno de Alfonso López Pumarejo (1942-1945). Por el contrario, los oficiales colombianos continuaron visitando Washington y asistiendo a las demostraciones de fuerza y de nuevos equipos que el Comando Sur de Estados Unidos realizaba en Panamá.

Las misiones estadounidenses se prorrogaron e incrementaron en su número de efectivos. Estudiantes y pilotos militares se trasladaron a Estados Unidos para recibir entrenamiento y adiestrarse en el manejo de los aviones que Colombia recibió en "préstamo y arriendo". La Armada y la Infantería del Ejército seleccionaron a sus mejores oficiales y suboficiales para cursos de especialización y estado mayor en Estados Unidos y en la zona del Canal. A ellos se unieron decenas de efectivos que cursaron estudios de aviación y mecánica de motores en Albrook Field, la más importante base aérea norteamericana para la defensa del Canal, construida en la ciudad de Balboa (Periódico El Espectador, 1943, septiembre 15 y 20). Poco después, las invitaciones se hicieron extensivas a cadetes o, incluso, a soldados colombianos (Periódico El Espectador, 1944, agosto 29).

En contra de lo que algunos analistas como Gerhard Drekonja (1982) consideran, ni el compromiso de Colombia con Estados Unidos, ni su política exterior, fueron absolutamente pasivos. Aunque Colombia no participó en la Segunda Guerra al nivel de México o Brasil, quienes enviaron tropas al Pacífico e Italia 
respectivamente, es claro que su decisión de apoyar a los aliados en caso de un ataque enemigo sobre Panamá fue más allá de la retórica.

Cierto es que Colombia no necesariamente se ubica a la cabeza de las decisiones en el hemisferio occidental y que, en la mayoría de las ocasiones, su apoyo se basó en las relaciones económicas o en sus aflicciones internas. Pero también es cierto que, a diferencia y algunas veces a despecho de los demás países latinoamericanos, la participación de Colombia en Corea y Suez refleja su papel definitivo en el respaldo a las políticas estadounidenses.

En materia diplomática, cinco hechos definen la consolidación del acercamiento de Colombia a los Estados Unidos al final de la Segunda Guerra e inicios de la Guerra Fría. En primer lugar, la clara política heredada de Eduardo Santos y continuada por la República Liberal, que puso a Colombia en el plano de lo que algunos autores denominan "subordinación activa"(Torres del Río, 1989). En segundo lugar, la redacción del "Acta de Chapultepec", que colocó a Colombia y a su Canciller, Alberto Lleras Camargo, como líderes continentales del sistema panamericano. En tercer término, el ingreso de Colombia como miembro fundador de la Organización de Naciones Unidas en 1945, en donde brindó importante soporte a las posiciones estadounidenses. No gratuita, ni pasivamente, Colombia hizo parte del Consejo de Seguridad, en dos ocasiones, durante los años cincuenta. En cuarto orden, el Tratado Interamericano de Asistencia Recíproca del cual fue Colombia un vivo autor e impulsor y que marcó la relación de los ejércitos latinoamericanos con su homólogo del norte. Finalmente, el papel fundamental de Colombia en la construcción de la Organización de Estados Americanos, con el significado de adhesión que tal entidad mantuvo.

Aunque pueda argumentarse que la política exterior de Colombia ha correspondido más a los gobiernos que al Estado, es un hecho que, de gobierno en gobierno, las relaciones con Estados Unidos permanecen prácticamente incólumes, se respetan los acuerdos sustanciales y se heredan sus aspectos más trascendentales. Al fin y al cabo, la economía influye en este caso al mundo de la política.

En materia militar, las citas panamericanas que exhortaron a la solidaridad continental en Lima y Panamá (1939), La Habana (1940) y Río de Janeiro (1942), abrieron el marco diplomático para los acuerdos de colaboración militar que empezaron a hacerse efectivos con la "Lend-lease Act" (Ley de préstamos y arriendo, 1941), que permitió la venta de armas y material de guerra a cualquier país cuya defensa se considerara vital para los intereses estadounidenses, y el Inter-American Defense Board (1942), que consintió e indujo el enlace de las Fuerzas Militares en todo el continente. Poco después, en 1947, el Tratado Interamericano de Asistencia Recíproca (TIAR) profundizó y legitimó el predominio militar de Estados Unidos en América Latina.

Sin embargo, para Colombia, luego de los acuerdos concretos y confidenciales a lo largo de la República Liberal y de las continuas visitas militares de misiones y 
alumnos a uno y otro país, el más significativo paso en el acercamiento de Colombia a Estados Unidos fue la tarea que Ospina Pérez depositó en las misiones estadounidenses para que formaran un Batallón de Infantería que sirviera como pilar para la difusión del proceder militar americano en el ejército colombiano. En adelante, el modelo militar de Estados Unidos se implementó de forma paulatina a través de la obra instructora de las misiones, la reorganización y modificación del sistema de Estado Mayor que el ejército heredaba aún de las misiones chilenas, o incluso el cambio de uniforme.

Como en la preguerra lo hizo el presidente liberal Eduardo Santos, en diciembre de 1951, bajo los fragores de la "Guerra Fría" ${ }^{1}$, Colombia rubricó un acuerdo informal y secreto que colocaba en manos de Estados Unidos la coordinación de las operaciones dentro de las rutas marítimas y aéreas para la protección del Canal de Panamá y el Mar Caribe en caso de agresión soviética, y reafirmó que cada país colocaría a disposición mutua los recursos militares, navales y aéreos indispensables para las operaciones militares "en defensa del hemisferio" 2 . Este fue, precisamente, un acuerdo que el gobierno conservador de Laureano Gómez (1950-1953) intentó utilizar para las adquisiciones de armas y tecnología dedicadas al control interno.

Meses después, en 1952, un acuerdo suscrito por el canciller colombiano Gonzalo Restrepo y el embajador norteamericano Capus M. Wainick, consolidó sobre la política de ambos Estados las decisiones en materia militar. Compuesto por once artículos, el acuerdo estableció el suministro de equipos, materiales y ayuda militar para "la defensa y la paz del hemisferio occidental", la permanencia indefinida de las misiones militares con carácter de personal diplomático y con las garantías correspondientes, el control del comercio con las naciones que amenazaran la seguridad continental, e incremento, por parte de Colombia, de su propia fuerza defensiva (Periódico El tiempo, 1952, 18 de abril).

No obstante, fue la Guerra de Corea el episodio que más acercó a los ejércitos de Colombia y Estados Unidos. Ella cimentó en los oficiales y soldados colombianos un "espíritu de cuerpo" que no habían sentido antes con respecto a sus similares norteamericanos. De hecho, el Batallón Colombia fue la primera unidad militar organizada conforme a los parámetros estadounidenses, luego de que el presidente Ospina solicitara esta labor a las misiones correspondientes, en 1947 (Lozano y Lozano, 1947).

En Corea, los militares colombianos formaron parte integral y subordinada de destacamentos americanos, se unieron a sus acciones, recibieron sus armas e instrucciones, compartieron el entrenamiento y exhibieron con orgullo las

\footnotetext{
${ }^{1}$ Obsérvese, al respecto, Bushnell, 1984 y Atehortúa, 2010.

${ }^{2}$ El texto original del acuerdo reposa en el Archivo de la Presidencia de la República bajo el título "SECRETO. Plan de los gobiernos de Colombia y de los Estados Unidos de Norteamérica para su defensa común". Embajada de Colombia, Washington, 31 de diciembre de 1951. ARCHIVO Presidencia de la República, 1954, Caja 843.
} 
condecoraciones que los altos oficiales norteamericanos otorgaron. En criterio de Rojas, pocos ejércitos como el colombiano contaban con las "invaluables enseñanzas de la guerra moderna y con la experiencia adquirida en distintos frentes" (Rojas, 1954, p. 243 -244).

Alberto Ruiz Novoa fue el primero de los oficiales colombianos que intentó sistematizar las experiencias obtenidas, con todas sus connotaciones de apego al ejército estadounidense (Ruiz Novoa, 1956). A su lado, importantes artículos publicados en la prensa nacional y en las revistas militares resaltaron desde las filas el significado que la guerra brindaba para la historia de los uniformados. Una vez más, algunos oficiales jóvenes enviaron oficios al ejecutivo destacando la colaboración norteamericana y manifestando sus deseos por profundizarla ${ }^{3}$.

Sobre el terreno, la influencia militar de Estados Unidos en Colombia se puso de presente con las enseñanzas que sobre la Guerra de Corea aplicaron los oficiales para la confrontación de las guerrillas en el país. La muerte en combate del cura guerrillero, Camilo Torres, se atribuye a la aplicación inicial de la táctica contraguerrillera aprendida en Corea. Las reformas orgánicas en la disposición interna de las Fuerzas Armadas, la Escuela de Lanceros, el Batallón de Policía Militar y el Batallón Antiaéreo de Armas Automáticas, constituyen una muestra sobre la acogida del modelo estadounidense. El armamento y la tecnología militar procedente de Estados Unidos se convirtieron, además, en una importante vía de relación y dependencia de las Fuerzas Militares colombianas. A ello se sumó la dependencia en órdenes distintos, incluyendo, desde luego, la ideológica. En las publicaciones de las Fuerzas Armadas colombianas desaparecieron paulatinamente las referencias y simpatías con respecto a los ejércitos de Europa y apareció la referencia y cita permanente del modelo estadounidense.

\section{CIVILES Y MILITARES. LOS LÍMITES DE LA AUTONOMÍA}

El golpe de Gustavo Rojas Pinilla en 1953 fue anunciado, consentido y propiciado por parte de la élite civil con el ánimo de recuperar para el Estado la funcionalidad de sus instituciones y de restaurar la legitimidad del sistema en medio de la violencia que azotaba al país. No fue una decisión de los militares ni tampoco un mandato de los americanos. Inspiradas y acosadas por la oposición civil, los militares asumieron el poder sin proyecto de gobierno, sin programa, sin perspectiva propia. Al rechazar lo que consideraron una afrenta contra su comandante y sus fuerzas, se encontraron de cara al gobierno porque los civiles a quienes lo ofrecieron no quisieron aceptarlo. A diferencia de sus congéneres del cono sur, los militares no buscaron el poder: se lo encontraron con las restricciones y los límites que le impusieron los civiles. Es más: lo recibieron y lo compartieron con ellos. No obstante, cuando Rojas empezó a alejarse de la dirigencia tradicional y de los partidos, y quiso convertir a su gobierno en una

\footnotetext{
${ }^{3}$ El documento escrito por el Teniente Coronel Hernando Medina, es un ejemplo de ello: "Si yo fuera el Comandante del Ejército", 1956. APR, Secretaría General, Fuerzas Militares, caja 2, carpeta 71, folio 287.
} 
dictadura de carácter más personal que incluso militar, no pudo resistir la arremetida de esa misma élite que implantó el Frente Nacional con la anuencia de la embajada estadounidense.

La caída de Rojas, el gobierno provisional de la Junta Militar y los profundos debates suscitados en el seno de los uniformados, marcó el reinicio de una metamorfosis útil para el Ejército y las Fuerzas Armadas. Al transferir poco a poco la administración del Estado a los civiles, al constatar el apoyo ciudadano al plebiscito de 1957 y observar los constantes peligros de corrupción y enfrentamiento interno por causa de la política, los militares abrazaron de nuevo sus preocupaciones de profesionalidad y asumieron los diversos desafíos planteados por las viejas enseñanzas de los chilenos, reestructuradas ahora bajo la influencia estadounidense.

La tentativa de golpe protagonizada el 2 de mayo de 1957 simboliza el último experimento de un reducido grupo de militares por restaurar su dominio sobre la élite civil y por recuperar la primacía en la administración del Estado. Su derrota permitió constatar la fortaleza histórica de los partidos en importantes sectores de las Fuerzas Militares, del mismo modo que los resultados electorales de la elección presidencial señalaron el deseo nacional de retornar a un régimen civil.

Pocos días después, el discurso del presidente electo, Alberto Lleras Camargo, fijó las pautas para una nueva época: la despolitización bipartidista de los militares y la consiguiente subordinación al Estado. Les brindó como contraprestación la posibilidad de adquirir una autonomía política relativa, dirigida al manejo del orden público y a la total independencia en la conducción de los asuntos militares, unida a ciertas prerrogativas institucionales y beneficios presupuestales. Si bien las Fuerzas Armadas no podrían decidir en adelante cómo gobernar a la nación, tampoco los políticos decidirían cómo manejar a las Fuerzas Armadas (Lleras Alberto, p. 213-214).

Aunque suene simplista, parece lógico pensar que el presidente de Colombia con mayor grado de confianza por parte de Estados Unidos en toda la historia del país, baluarte sin igual en la política norteamericana del final de los años cuarenta con respecto a Latinoamérica, actor principal en la redacción del TIAR, en la creación de la OEA y del posterior rechazo a la revolución cubana, delegara en los militares, cuya formación y orientación era delineada por las Fuerzas Armadas de los Estados Unidos, toda responsabilidad frente al manejo del orden público en un momento en que la Guerra Fría se colocaba al orden del día.

Los partidos y sus dirigentes, entre tanto, estaban más interesados en la construcción de maquinarias electorales locales y regionales, en el usufructo del clientelismo o en el engranaje general del nuevo régimen. De modo que, si los militares recibían instrucción para ejercer en forma debida sus funciones, bien podrían encargarse de la elaboración de planes estratégicos relacionados con el orden interno y el nuevo esquema de "Seguridad Nacional". Así, más que un deseo de los militares por intervenir en las políticas del Estado o de buscar su 
autonomía en el manejo del orden público, fue la negligencia de los civiles y un pacto inspirado en la profunda confianza sobre los militares bajo influencia estadounidense, lo que facilitó el nuevo papel de las Fuerzas Armadas, con el único límite de que sus actos no amenazaran el statu-quo del gobierno en manos de los civiles, o las estrategias clientelistas y electorales de los partidos ${ }^{4}$.

De esta manera, en la alborada del Frente Nacional, los militares colombianos, bajo la decisiva influencia estadounidense, asumieron la seguridad del Estado sobre la base del anticomunismo propiciado por la Guerra Fría y con la aquiescencia de la dirección civil. Poco a poco, la "Defensa Nacional" pregonada por los chilenos en la madrugada del siglo XX, cedió el paso a la "Seguridad nacional" como nueva categoría de seguridad adoptada por Estados Unidos y Suramérica en los álgidos tiempos del mundo bipolar. En materia militar, Colombia convirtió a la estrella austral en referente histórico y de formación en el pasado, y fijó su mirada, definitivamente, en la estrella polar.

Las Misiones Chilenas que impulsaron la Reforma Militar en 1907, que crearon la Escuela Militar, la Escuela Naval y la Escuela Superior de Guerra, que sembraron las primeras semillas de profesionalidad pero fracasaron finalmente ante el empuje de los partidos, del clientelismo y del gamonalismo regional, pasaron a la historia como ocurrió de manera aún más silenciosa con la Misión Suiza que intentó en vano la transformación del Ejército al final de los años veinte ${ }^{5}$. Su lugar, con eficacia y establecido compromiso, fue ocupado por las misiones estadounidenses que, de diverso tipo, empezaron a llegar al país cuando, al mismo tiempo, los militares colombianos viajaron a Estados Unidos y Panamá para recibir en las academias norteamericanas o en la Escuela de las Américas, la instrucción que la nueva época les deparaba.

\section{COLOMBIA - ESTADOS UNIDOS: EFECTOS INICIALES DE LA INFLUENCIA}

La influencia militar estadounidense puede constatarse a través de dos efectos: el proceso de modernización iniciado bajo la égida norteamericana y la ayuda financiera que, en materia militar, Estados Unidos proporcionó a Colombia.

\subsection{El proceso de modernización bajo nuevos esquemas}

Una de las más importantes influencias que el modelo militar estadounidense aplicado en Corea desarrolló en Colombia, se verifica con la creación de la "Escuela de Lanceros". Según la versión oficial, la unidad fue construida como parte de una reforma general aplicada a los sistemas militares del ejército colombiano, obligado a impulsar transformaciones en su seno ante las arremetidas

\footnotetext{
${ }^{4}$ Un ejemplo importante del papel de los militares y de sus límites lo constituye el "Plan Lazo" elaborado por el General Alberto Ruiz Novoa, cuyos avatares se enuncian en diversas fuentes bibliográficas como Francisco Leal Buitrago, El oficio de la guerra y César Torres del Río, Fuerzas Armadas y Seguridad Nacional.

${ }^{5}$ Obsérvese Atehortúa, 2009.
} 
del orden público interno y las apreciaciones sobre la lucha internacional contra el comunismo.

Ante la aparición en el plano nacional de modalidades de lucha con tácticas de combate irregular, el comando del ejército decidió enviar en 1955 una comisión de estudios a la Escuela de Rangers del Ejército de Estados Unidos, en Fort Benning. Dicho curso fue considerado como "el más indicado para la adquisición de la técnica de combate irregular, toda vez que se les capacitaría como comandantes de pequeñas unidades para el cumplimiento de misiones especiales" 6 . A su regreso, mediante Decreto No. 3155 del 6 de diciembre de 1955, y con la asesoría del capitán Ralph Puckett, la Escuela inició en la localidad de El Nilo (Cundinamarca), su primer curso de entrenamiento ${ }^{7}$. La preparación para la conducción de "pequeñas patrullas de infantería en toda clase de circunstancias y situaciones difíciles", se asumió como objetivo principal de la Escuela.

En un principio, los alumnos asistentes a la Escuela fueron oficiales subalternos, suboficiales de reconocida trayectoria, y excepcionalmente soldados. La idea era convertirlos en instructores capacitados que pudieran replicar su aprendizaje en las diferentes unidades militares del país, lo que lograron incluso en términos de subcultura. Bajo el esquema ranger, el ejército de Estados Unidos propuso a sus miembros en Corea una relación básica de apoyo y seguridad mutua: la relación soldado-soldado que, como grupo social mínimo y cotidiano, construyó en la táctica un sistema de movilidad por parejas apto para un territorio hostil y desconocido. Diseminado en Colombia a través de los lanceros, el término "lanza" se convirtió en sinónimo de amigo incondicional y fiel en las filas del ejército.

En 1959, las "compañías de lanceros" entraron a formar parte de diversas unidades militares, concebidas como una organización más ágil y flexible que permitió éxitos en la lucha antisubversiva. A partir de esa fecha, el Comando del Ejército determinó la creación de unidades aún más pequeñas pero altamente entrenadas, con una movilidad superior y con preparación de unidades especializadas de localizadores y contraguerrillas.

En 1962, el ejército concluía que "la guerra de guerrillas" sería "una situación que tendremos que afrontar por tiempo imprevisible" y que los Estados Unidos se preparaban "para luchar en una guerra de guerrillas" como producto de la táctica comunista para "adueñarse del poder". Se propuso, por consiguiente, "un cambio de mentalidad para considerar la instrucción individual básica y la de lanceros como algo primordial" y "obligatorio en todas las unidades tácticas, extendiéndola a todas las fuerzas militares y de policía (Revista del Ejército, Volumen II, 1962, p. 131-135).

\footnotetext{
${ }^{6}$ Ver http://www.ejercito.mil.co/index.php?idcategoria=92

${ }^{7}$ Escuela de Lanceros, 1957. Una importante síntesis sobre la importancia de la Escuela de Lanceros como unidad representativa del proceso de adscripción militar a los Estados Unidos puede encontrarse en: Saúl Mauricio Rodríguez, 2006.
} 
Como resultado de la promovida necesidad y efectividad de los lanceros, las fuerzas militares de varios países latinoamericanos solicitaron asistir a los cursos contraguerrilleros dictados en Colombia, los cuales empezaron a programarse en 1966 como versión internacional, con el aval de la Escuela de las Américas. Allí se impulsó, igualmente, la idea de la autodefensa de la población civil como una forma de "organizarla para neutralizar a los violentos... mientras llega el ejército a relevarlos y a desarrollar una acción definitiva" (Revista del Ejercito, Volumen II, 1962, p. 41-44).

Otro aporte concreto de la influencia estadounidense se expresó en la creación del primer Batallón de Policía Militar y del primer Batallón Antiaéreo de Armas Automáticas. La creación del Batallón de Policía Militar fue asignada a la misión encabezada por el capitán Robert Brenner, complementada por oficiales preparados en el "Curso avanzado de policía militar" en Camp Gordon (Georgia). Su objetivo era el control de manifestaciones públicas bajo el estudio de prácticas centradas en "psicología de masas", "formación antimotines", "empleo de gases y elementos dispersores", y "utilización de fuerza controlada". La Policía Militar se convirtió, en su primer momento, en un cuerpo de élite sumamente respetado en las propias filas. Aunque su participación en el frustrado golpe del 2 de mayo originó su suspensión temporal, cuatro meses después se restableció el propósito a plenitud.

En concepto de Alberto Ruiz Novoa, la aplicación de esta experiencia coreana en Colombia permitiría ampliar los propósitos de la Policía Militar a la dirección del tránsito en las calles citadinas, el trato adecuado de civiles en áreas militares, captura de desertores, o incluso labores policivas en áreas de operaciones militares y combate a la corrupción interna (Ruiz Novoa, 1956, p. 300-301). La creación del Batallón Antiaéreo se ordenó a través de Decreto Número 135 de 1953, como anexo de la Escuela de Artillería y a cargo de la Misión Militar estadounidense, como "parte de los compromisos emanados del Convenio Bilateral de Ayuda Militar" (Bermúdez).

Algunos documentos históricos permiten deducir, igualmente, cierta vinculación de la CIA y del FBI con la creación del "Servicio de Inteligencia Colombiano" (SIC), a solicitud del presidente Gustavo Rojas Pinilla. Al menos, el salario acordado por el embajador de Colombia en Washington con un agente de la CIA para la organización del SIC, fue por US $\$ 10.000$ anuales "más los gastos". La CIA serviría como "órgano de consulta del SIC" y se comprometía a "suministrar el personal competente para cualquier trabajo especial u ocasional" que fuera requerido, sin costo adicional para Colombia 8 .

El armamento y la tecnología militar estadounidense se convirtieron, también, en una importante vía de relación y dependencia de las Fuerzas Militares colombianas con respecto a su vecino del norte. En 1950, según el Ministro de

\footnotetext{
${ }^{8}$ Informe del Embajador Eduardo Zuleta al Presidente Gustavo Rojas Pinilla. Washington, octubre 21 de 1953. APR, 1953, caja 792.
} 
Guerra de entonces, la Dirección de Material de Guerra había realizado entre sus actividades importantes el "cambio de calibre de las armas menores, adaptándolo al calibre de los Estados Unidos" y adquiría, en adelante, nuevas armas según las especificaciones americanas (Bernal, 1951, p. 78-79). En 1954, la transformación operaba sobre las armas largas con los calibres punto 30 y punto 50 , con las cuales se siguió la tendencia del armamento que llegó al país a través del Batallón Colombia en Corea y de los acuerdos de asistencia militar.

A pesar de las dilaciones y negativas de Estados Unidos para suministrar armas a Colombia en medio de la violencia interna y del gobierno de Rojas, Colombia reestructuró en 1949 un pequeño escuadrón de aviones F-47 y otro de bombardeo con aviones B-25, utilizados por Estados Unidos para el ataque a Tokio en la Segunda Guerra Mundial. Durante el gobierno de Rojas recibió seis aviones de combate Lockheed tipo jet T-33르, seis Jet Sabre F-86, algunos Mentor para entrenamiento de pilotos, bombarderos B-26 y aviones de carga C-54 que actualizaron el andamiaje de la Fuerza Aérea (Forero, 1964).

En el plano arquitectónico la influencia estadounidense empezó a sentirse con la construcción del "Salón de la Defensa" del Centro de Infantería de la Escuela Militar de Cadetes, a semejanza del existente en Fort Benning, con la asesoría del Mayor Jesse Ugalde, miembro de la Misión Militar de Estados Unidos en 1949 y 1950 (Álvarez \& Giraldo, 1997, p. 87).

\subsection{La ayuda financiera}

La ayuda financiera estadounidense en América Latina se expresó, en los años sesenta y principios de la década de los setenta, a través de cuatro fuentes principales: la Agency for Internacional Development (AID) con donaciones o créditos, los créditos de la Organización Panamericana de la Salud (OPS), auxilios especiales aprobados por el Congreso de los Estados Unidos y el Programa de Asistencia Militar.

Al término de la Segunda Guerra Mundial y antes de la Guerra de Corea, Colombia no fue uno de los países latinoamericanos que más ayuda estadounidense recibió. En ambos períodos ocupó el lugar once, por debajo de países con menor población y extensión como Paraguay, Costa Rica, Ecuador, Perú y Bolivia. 
Cuadro No. 1

Ayuda exterior estadounidense

Donaciones antes y después de Corea

(Cifras en millones de dólares)

\begin{tabular}{|c|l|r|r|c|}
\hline Posición & \multicolumn{1}{|c|}{ País } & Antes de Corea & Después de Corea & Posición \\
\hline 1 & México & 82.616 .000 & 14.755 .000 & 1 \\
\hline 2 & Brasil & 9.241 .000 & 5.111 .000 & 2 \\
\hline 3 & Chile & 4.378 .000 & 2.553 .000 & 7 \\
\hline 4 & Perú & 3.988 .000 & 4.394 .000 & 3 \\
\hline 5 & Guatemala & 3.947 .000 & 655.000 & 15 \\
\hline 6 & Haití & 2.723 .000 & 1.840 .000 & 10 \\
\hline 7 & Ecuador & 2.430 .000 & 2.720 .000 & 4 \\
\hline 8 & Bolivia & 2.343 .000 & 2.567 .000 & 6 \\
\hline 9 & Paraguay & 2.221 .000 & 2.694 .000 & 5 \\
\hline 10 & Costa Rica & 1.947 .000 & 2.504 .000 & 8 \\
\hline 11 & Colombia & 1.721 .000 & 1.599 .000 & 11 \\
\hline 12 & Venezuela & 1.349 .000 & 426.000 & 18 \\
\hline 13 & El Salvador & 1.146 .000 & 1.131 .000 & 14 \\
\hline 14 & Uruguay & 834.000 & 596.000 & 16 \\
\hline 15 & Honduras & 618.000 & 1.470 .000 & 12 \\
\hline 16 & R. Dominicana & 499.000 & 588.000 & 17 \\
\hline 17 & Cuba & 446.000 & 296.000 & 19 \\
\hline 18 & Panamá & 397.000 & 2.148 .000 & 9 \\
\hline 19 & Nicaragua & 396.000 & 1.215 .000 & 13 \\
\hline 20 & Argentina & 198.000 & & 20 \\
\hline
\end{tabular}

Fuente: Chávez, José María. Informe sobre la ayuda exterior de Estados Unidos, 1953. APR, 1954, Caja 827. Citado por César Torres del Río (2000, p. 29).

No obstante, Colombia sí fue uno de los países más beneficiados con la ayuda militar estadounidense. Entre 1953 y 1966, Brasil ocupó el primer lugar, pero tenía un ejército cuatro veces más grande que el colombiano en un territorio seis veces mayor. Comparativamente, Chile fue el más privilegiado: ocupaba el segundo lugar en apoyo financiero con cuerpos militares iguales en cantidad a los de Colombia en un territorio mucho menor, con la mitad de población. Aunque la ayuda se redujo durante la dictadura de Rojas, Colombia disputó a partir de 1958 el tercer lugar con Perú. Luego de Argentina, la ayuda ofrecida por Estados Unidos a los demás países del área era realmente débil. 
Cuadro No. 2

Asistencia militar estadounidense para América Latina

Años Fiscales 1953-1966

(Cifras en millones de dólares)

\begin{tabular}{llr}
\hline $\mathbf{N}^{\circ}$. & \multicolumn{1}{c}{ País } & $\begin{array}{r}\text { Cantid } \\
\text { ad }\end{array}$ \\
\hline 1 & Brasil & 374.1 \\
\hline 2 & Chile & 143.4 \\
\hline 3 & Perú & 134.3 \\
\hline 4 & Colombia & 102.3 \\
\hline 5 & Argentina & 87.6 \\
\hline 6 & Venezuela & 66.8 \\
\hline 7 & Ecuador & 52.9 \\
\hline 8 & Uruguay & 44.5 \\
\hline 9 & R. & 19.6 \\
& Dominicana & \\
\hline 10 & Bolivia & 17.6 \\
\hline 11 & Cuba & 16.1 \\
\hline 12 & Guatemala & 13.4 \\
\hline 13 & Nicaragua & 9.4 \\
\hline 14 & Paraguay & 8.3 \\
\hline 15 & México & 6.7 \\
\hline 16 & Honduras & 6.1 \\
\hline 17 & El Salvador & 5.3 \\
\hline 18 & Haití & 4.5 \\
\hline 19 & Panamá & 2.3 \\
\hline 20 & Costa Rica & 2.0 \\
\hline & & $\mathbf{1 . 1 3 5 ,}$ \\
& Total & $\mathbf{8}$ \\
\hline
\end{tabular}

Fuente: The Latin American Military. Estudio preparado para el Congreso Norteamericano por Edwin Lieuwen. Washington: Government Printing Office, 1967. p. 36. Cuba sólo recibe ayuda hasta 1961 y se suspende la de Haití en 1962.

En los años siguientes, aunque las informaciones son parciales, la tendencia es similar. Sin tomar en cuenta la ayuda militar por créditos, el aporte americano a través del Programa de Asistencia Militar destina para Colombia, entre 1964 y 1968, más del once por ciento del total asignado a toda Latinoamérica y alcanza incrementos importantes y superiores al $16 \%$ en 1968. 


\section{Cuadro No. 3 \\ Ayuda militar esadounidense para Colombia y América Latina 1964-1968}

(Cifras en millones de dólares)

\begin{tabular}{|c|c|c|c|c|c|c|}
\hline Año & $\begin{array}{c}\text { MAP } \\
\text { Colombia }\end{array}$ & $\begin{array}{c}\text { MAP } \\
\text { Latinoamérica }\end{array}$ & $\%$ & $\begin{array}{c}\text { Ayuda } \\
\text { AID }\end{array}$ & $\begin{array}{c}\text { Créditos } \\
\text { AID/OPS }\end{array}$ & $\begin{array}{c}\text { Total Ayuda } \\
\text { Norteamericana } \\
\text { para Colombia }\end{array}$ \\
\hline 1964 & 6.20 & 52.10 & 11.9 & 0.69 & 0.34 & 7.23 \\
\hline 1965 & 5.70 & 55.90 & 10.1 & 0.24 & 0.81 & 6.75 \\
\hline 1966 & 3.30 & 58.40 & 5.6 & 0.67 & 1.79 & 5.76 \\
\hline 1967 & 7.90 & 59.10 & 13.3 & 0.87 & 0.50 & 9.27 \\
\hline 1968 & 12.20 & 72.80 & 16.7 & 0.10 & 0.34 & 12.64 \\
\hline Total & $\mathbf{3 5 . 3 0}$ & $\mathbf{2 9 8 . 3 0}$ & $\mathbf{1 1 . 8}$ & $\mathbf{2 . 5 7}$ & $\mathbf{3 . 7 8}$ & $\mathbf{4 1 . 6 5}$ \\
\hline
\end{tabular}

Fuente: Muri, Willy. L'armée colombienne: étude d'une institution militaire dans ses rapports avec la société en transition, 1930-1974. Thèse de $3^{\mathrm{e}}$. cycle. IHEAL. Paris, 1975. MAP: Military Aid Program.

El número de militares colombianos entrenados por Estados Unidos es igualmente considerable. Entre 1950 y 1970, al menos 4.629 militares de Colombia pasaron por las Escuelas estadounidenses en su propio territorio o en las bases ubicadas en Panamá. La cifra, sin embargo, puede ser mayor. Ha sido calculada con datos aislados del Departamento de Defensa, con los "Military Assistance Facts", una publicación periódica de los militares americanos, y con el "Nacla's Hand Book", no con los archivos del Ministerio de Defensa colombiano. La cifra tampoco incluye a los militares que obtuvieron instrucción gracias a su participación en la guerra de Corea, ni a los oficiales de policía. Otras fuentes mencionan que desde 1956 hasta 2003, un total de 9.886 militares colombianos se graduaron en la Escuela de las Américas, muchos más que aquellos de Chile (5.347), de Nicaragua (4.318) o de Bolivia (3.878) y más del diez por ciento de la cifra general latinoamericana $(61.000$ aproximadamente) ${ }^{10}$.

Además de lo anterior, no pocos miembros de las Fuerzas Militares recibían instrucción directa en Colombia. A diciembre de 1966, el informe rendido por Edwin Lieuwen al Senado de Estados Unidos menciona 34 oficiales americanos asignados a misiones militares en Colombia. La cantidad sólo es superada por Brasil, en donde se encuentran 53 oficiales, y por Venezuela, quien cuenta con 36. Pero, para observar la magnitud de las cifras arriba calculadas, debe tenerse en cuenta que el número total de efectivos de las Fuerzas Militares de Colombia, hasta 1968, no fue más allá de 50.000 unidades.

\footnotetext{
${ }^{9}$ Las fuentes en mención pueden consultarse en la Biblioteca del Congreso Norteamericano.

${ }^{10} \mathrm{http} / / /$ www.viejoblues.com/escuelamericas.htm; consultado el 17 de febrero de 2010. Según los datos recopilados por School of the Americas Watch, el número total de graduados en la Escuela asciende a 60761. Cf. http://www.soaw.org/grads/: consultado el 17 de febrero de 2010.
} 
Igualmente significativo resulta el examen a las posiciones claves que en el ejército y el gobierno colombianos, durante el Frente Nacional, ocuparon los oficiales que se formaron y/o desempeñaron funciones previas en Estados Unidos. Entre aquellos que estuvieron en Corea, uno fue ministro de defensa, otro fue comandante del Ejército, dos lo fueron de la Marina, dos llegaron a ser jefes del Estado Mayor Conjunto, diecisiete comandantes de Brigada, dos fueron directores de la Escuela Militar y uno de la Escuela Naval ${ }^{11}$.

Un destino similar siguió la mayoría de los oficiales colombianos destinados en Misiones a Estados Unidos. Varios de los asignados a la Junta Interamericana de Defensa 0 adjuntos a la Embajada Colombiana en Washington, por ejemplo, pasaron luego por el Ministerio de Defensa, por el Comando del Ejército, de la Fuerza Aérea y de la Marina, por el Estado Mayor Conjunto, por la Dirección de la Escuela Superior de Guerra, o incluso de la Industria Militar. En la práctica, de la misma manera como se acostumbró a que los presidenciables pasaran primero por la Embajada de Colombia en Estados Unidos, los altos oficiales de las Fuerzas Militares pasaban previamente por comisiones en el país del norte ${ }^{12}$.

\section{FUENTES}

The Latin American Military. Estudio preparado para el Congreso Norteamericano por Edwin Lieuwen. Washington: Government Printing Office, 1967

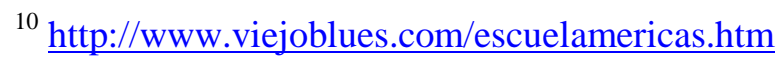

\section{BIBLIOGRAFÍA}

Álvarez, Víctor (Brigadier General) \& Hernán Giraldo (Mayor). (1997). Escuela Militar de Cadetes General José María Córdova. Noventa años de historia. Bogotá: Litografía Arco.

Atehortúa, Adolfo. (1994). Estado y Fuerzas Armadas en Colombia. Bogotá, Tercer Mundo, Universidad Javeriana Cali.

----. (2009). Construcción del Ejército Nacional en Colombia, 1907-1930. Reforma Militar y Misiones extranjeras. Medellín: La Carreta.

\footnotetext{
${ }^{11}$ La relación, sin embargo, es sólo tentativa. Se ha efectuado de acuerdo con la lista del "Batallón Colombia" publicada por Ramiro Zambrano en "Siluetas para una historia" (Suplemento de la Revista del Ejército, vol. 8, No. 29, julio de 1967) y registrando los nombres obtenidos para algunos cargos militares importantes hasta 1974.

${ }^{12}$ Algunos nombres podrían citarse a título de sustento. Ellos son, entre otros, los generales Ayerbe Chaux, Calderón Molano, Camacho Leyva (Alberto), Currea Cubides, Fajardo Pinzón, Gutiérrez Ospina, Herrera Calderón, Mejía Valenzuela, Ospina Navia, Rincón Puentes, Rubiano Groot, Sánchez O’Donohuel, Valencia Tovar, y los almirantes Parra Ramírez y Piedrahita Arango.
} 
----. (2010). Partidos, Violencia y Ejército (1934-1957). Bogotá: Universidad Militar Nueva Granada.

Bermúdez, Julián. Escuela de Artillería Colombiana, 1936-2000. S.p.i.

Bernal, José María. (1951). Memoria del Ministerio de Guerra 1950-1951. Bogotá: Imprenta del Comando General de las Fuerzas Militares.

Bushnell, David. (1984). Eduardo Santos y la Política del Buen Vecino. Bogotá, El Áncora.

Drekonja, Gerhard. (1982). Colombia: Política Exterior. Bogotá: Universidad de los Andes.

Escuela de Lanceros. (Septiembre de 1957). "Reseña histórica sobre las unidades rangers". En, Revista Militar. Tomo III, Número 9.

Forero, José Ignacio. (1964). Historia de la aviación en Colombia. Bogotá: Aedita editores Ltda.

Leal Buitrago, Francisco. (1994). El oficio de la guerra. Bogotá: Tercer MundoIEPRI.

----. (2002). La seguridad nacional a la deriva. Del Frente Nacional a la posguerra fría. Bogotá: Alfaomega, Ceso-Uniandes, FLACSO-Sede Ecuador.

Lozano y Lozano, Fabio. (1947). Memoria del Ministro de Guerra al Congreso Nacional, 1947. Bogotá: Imprenta del Ministerio de Guerra.

Lleras, Alberto. (Edición sin fecha). Sus mejores páginas. Discurso pronunciado en el Teatro Patria, ante oficiales de la Guarnición de Bogotá. Lima: Editora Latinoamericana, 2o. Festival del libro colombiano, Biblioteca Básica de Cultura.

Medina, Hernando. "Si yo fuera el Comandante del Ejército". Bogotá, diciembre 12 de 1956. APR, Secretaría General, Fuerzas Militares, caja 2, carpeta 71, folio 287.

Rodríguez, Saúl. (2006). La influencia de los Estados Unidos en el Ejército colombiano, 1951-1959. Medellín: La Carreta, Universidad Nacional.

Rojas, Gustavo. (1954). "Bienvenida a la Patria". Saludo del General Rojas al regreso del Batallón Colombia, noviembre 30 de 1954. En, Mensajes y discursos. Bogotá: Imprenta Nacional.

Ruiz Novoa, Alberto. (1956). Enseñanzas militares de la campaña de Corea aplicables al Ejército de Colombia. Bogotá: Antares. 
Torres del Río, César. (Julio-agosto de 1989). "El presidente Santos y la nueva práctica de la política exterior de Colombia". En, Documentos Ocasionales. Bogotá: Centro de Estudios Internacionales de la Universidad de los Andes.

----. (2000). Fuerzas Armadas y Seguridad Nacional. Bogotá: Planeta.

\section{Prensa}

El Espectador. (1943, 15 y 20 de septiembre).

El Tiempo. (1952, 18 de abril).

---- . (1944, 29 de agosto).

Revista del Ejército. (1962). Volumen II. p. 41-44.

Recibido: Noviembre 5 de 2010

Aprobado: Mayo 9 de 2011 